\title{
What You Feel Is What I Do: A Study of Dynamic Haptic Interaction in Distributed Collaborative Virtual Environment
}

\author{
Sehat Ullah ${ }^{1}$, Xianging Liu ${ }^{3}$, Samir Otmane ${ }^{1}$, \\ Paul Richard ${ }^{2}$, and Malik Mallem ${ }^{1}$ \\ 1 IBISC Laboratory, University of Evry, \\ 40 rue de pelvoux 91020, France \\ sehat.ullah@ibisc.univ-evry.fr \\ http://www.ibisc.univ-evry.fr \\ 2 LISA Laboratory, University of Angers, \\ 62 av Notre Dame du lac 49045, France \\ 3 Tokyo Institute of Technology, Japan
}

\begin{abstract}
In this paper we present the concept of "What You Feel Is What I Do (WYFIWID)". The concept is fundamentally based on a haptic guide that allows an expert to control the hand of a remot trainee. When haptic guide is active then all movements of the expert's hand (via input device) in the 3D space are haptically reproduced by the trainee's hand via a force feedback device. We use haptic guide to control the trainee's hand for writing alphabets and drawing geometrical forms. Twenty subjects participated in the experiments to evaluate.
\end{abstract}

Keywords: Haptic guide, CVE, Virtual reality, Human performence.

\section{Introduction}

The Collaborative Virtual Environments(CVEs) have attained a growing interest from the research community since the last few years. A CVE is a computer generated world that enables people in local/remote locations to interact with synthetic objects and representations of other participants within it. The applications domain for such environments can be in military training, telepresence, collaborative design and engineering, entertainment and education. Interaction in CVEs can be categorized in the following way, also discussed in [36.

The environments that allow multiple users, but only one user is active at a time and is capable to interact and/or manipulate the objects. The others remain passive and wait their turn. The environments where users perceive the copresence through avatars but each user independently interacts with the objects. Any change to the attribute of an object or scene is visible to all collaborators. The environments where two or more users can manipulate the same object. This types of manipulation can be asynchronous or synchronous. The synchronous/ concurrent manipulation is also termed as cooperative manipulation.

J.A. Jacko (Ed.): Human-Computer Interaction, Part II, HCII 2011, LNCS 6762, pp. 140-147, 2011. (C) Springer-Verlag Berlin Heidelberg 2011 
CVEs can be used for learning purposes. For example, if one or more users watch, how a particular task is performed parformed by the expert in the Virtual Environment (VE). This is called learning by observation but it may not be so much effective without haptic feedback because it plays a vital role in learning or transferring motor skills [19 18 16 17]. Most of these systems are single user and use the mechanism of record and play of force and position values. Similarly, force feedback has also been used in both single user and collaborative VEs in order to impart the users more realism and increase their performance but dynamique haptic guidence in 3D distributed CVEs has rarely been investegated.

This section is followed by the related work. Section 3 presents the arhitecture of the VE. Secion 4 presents the model for the haptic guide and its evaluation. Conclusion is presented in section 5 .

\section{Related Work}

Basdogan et al. have investigated the role of force feedback in cooperative task. They connected two monitors and haptic devices to a single machine [1]. Similarly, sallnas et al. have reported the effect of force feedback over presence, awareness and task performance in a CVE. They also connected two monitors and haptic devices to a single host [11]. But all these systems use force feedback only for realism and not for guidance in collaboration. Other important works that support the cooperative manipulation of objects in a VE include [45] but all these systems require heavy data exchange between two nodes to keep them consistent. Haptic guides have successfully been used for 3D object selection in large scale VEs [13. McSig is a multimodal teaching and learning environment for visually-impaired students to learn character shapes, handwriting and signatures collaboratively with their teachers. It combines haptic and audio output to realize the teacher's pen input on a specialized sheet for visually disable people $[8$. Here both teacher and student connect to the same computer. CHASE (Collaborative Haptics And Structured Editing), is a synchronous structured drawing tool. It provides telepointers and allows users to simultaneously work on a large canvas while each maintaining a separate view of it [15. In [7 a virtual environment that allows two users to collaboratively sculpt from remote locations, has been presented. Here haptic feedback is used to render the tool's pressure on the clay and to avoid the simultaneous editing of a vertex. Similarly Chan et al. 22 have reported the use of vibro-tactile cues to facilitate turntaking in an environment that support collaboration but only one user remains in control and has the rights to manipulate objets at a particular instant. Virtual Fixtures (VFs) formalism has been presented in [12] and mechanics based characterization and design guidelines have been reported in [9].

Similarly, Chellali et al. have also investigated the learning of an insertion task through haptic feedback 20. In this study, they allowed two users to see the virtual world using two desktop displays with the same computer while sending the position values of the master Virtuose to the slave through network. The system that we propose is distributed and guidance is not limited to a single task. 


\section{Dynamique Haptic Interaction}

\subsection{System Architecture}

Here we present our system that allows an expert to haptically guide the hand of non-expert in the 3D space. The two users (expert and non-expert) use two separate machines connected through local area network. Our application that allows collaboration is installed on each of these machines [14. Whenever the applications are launched on both systems and network connection is successfully established between them, each user will see the VE on their screens. The VE has room a like structure to give the 3D perception. This environment contains two spheres each representing a user. Each user controlls the movements of his/her corresponding sphere through a haptic device.

\subsection{Hapitc Guide}

The name "What You Feel Is What I Do" is given to the concept because of the manner the force acts on the trainee. When all the conditions for haptic guide's activation are satisfied then all movements by the expert's input device in the 3D space are haptically reproduced by the trainee's hand via a force feedback device (Phantom and SPIDAR in our case). More specifically any 3D motion done by the expert is reproduced and haptically felt by the trainee or the person using the second machine. The spatial information (position) of the two input devices are mutually exchanged and visualised on both machines but the force is only locally calculated on the trainee's machine.

Referring to the figure 1, $U e$ and $U t$ represent the spheres of the expert and trainee respectively. Similarly $r_{e}$ and $r_{t}$ are the radii of the spheres representing the expert and trainee respectively. The distance between the two is denoted by $d$.

$$
\begin{aligned}
P_{u e} & =\left(X_{u e}, Y_{u e}, Z_{u e}\right) \\
P_{u t} & =\left(X_{u t}, Y_{u t}, Z_{u t}\right)
\end{aligned}
$$

Here $P_{u e}$ and $P_{u t}$ represent the positions of the expert's and trainee's sphere respectively. In order to activate the guide, the expert envoks an event for example pressing a button of the phantom stylus. This event will change the color of the expert's sphere on both machines. The second condition that must be true for guide activation is:

$$
d \leq r_{e} * r_{t}
$$

The guiding force which attracts the trainee's sphere towards the centre of the expert's sphere is calculated in the following way,

$$
F=K *\left(\left(X_{u e}-X_{u t}\right)_{x}+\left(Y_{u e}-Y_{u t}\right)_{y}+\left(Z_{u e}-Z_{u t}\right)_{z}\right)
$$

Where $K$ is a constant and was experimentally set in order to have a smooth but not very rigid force. This guide can be used for childern to learn alphabets and digits of various languages (i.e english, arabic etc.), geometrical forms and 


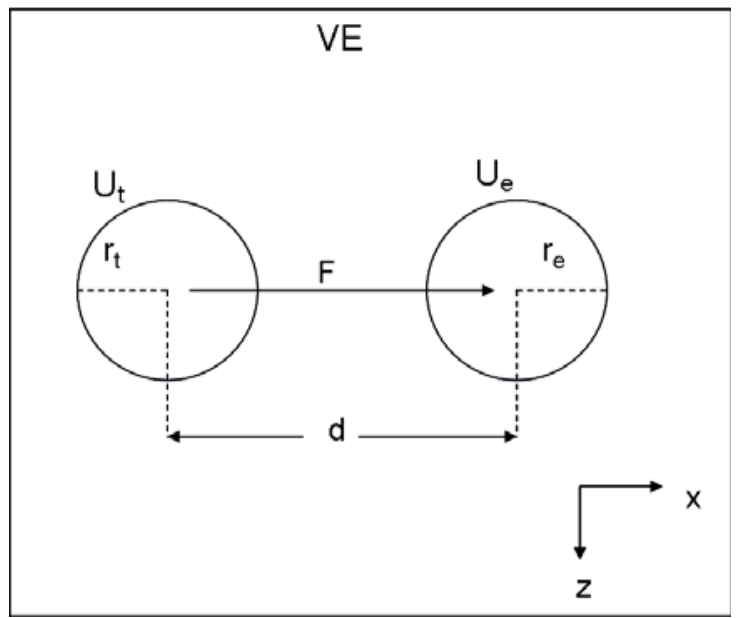

Fig. 1. Illustraion of haptic guide model (top view of $V E$ )

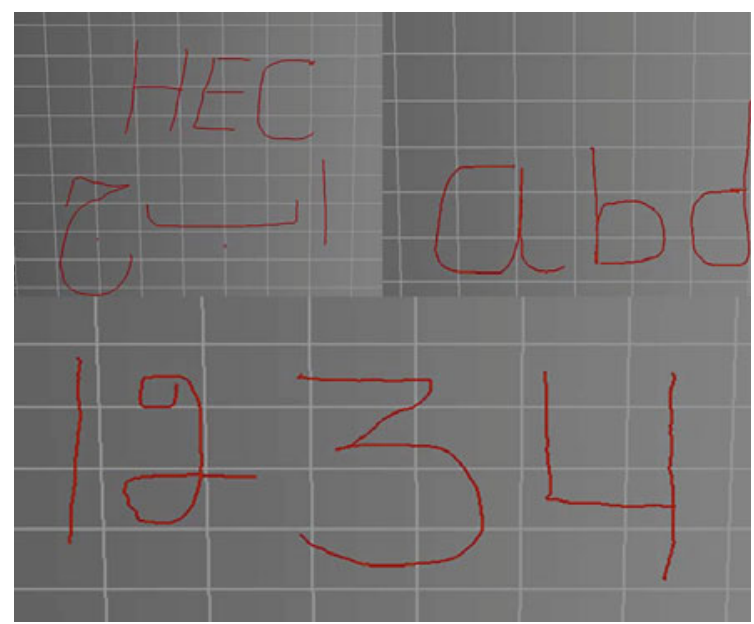

Fig. 2. Writing alphabets and digits with the help of the haptic guide

drawings (see figure 2) in the supervision of an expert (teacher). The guide does not require to record the characters, digits or geometrical forms in advance.

The concept was implemented using two different configurations. In the first implementation, we used two desktop computers connected through Local Area Network (LAN). Each computer was equipped with a Phantom omni. In the second implementation two large scale platforms were used. Both platforms are equiped with SPIDAR ( Space Interface Device for Artificial Reality) (see figure 3) 21 and connected through LAN. Here, SPIDAR-GH and SPIDAR-GM are used for $3 \mathrm{D}$ tracking as well as for force rendering. Where SPIDAR-GH 


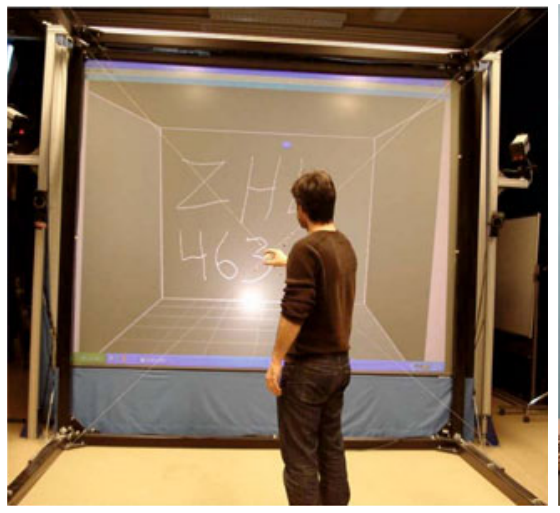

(a)

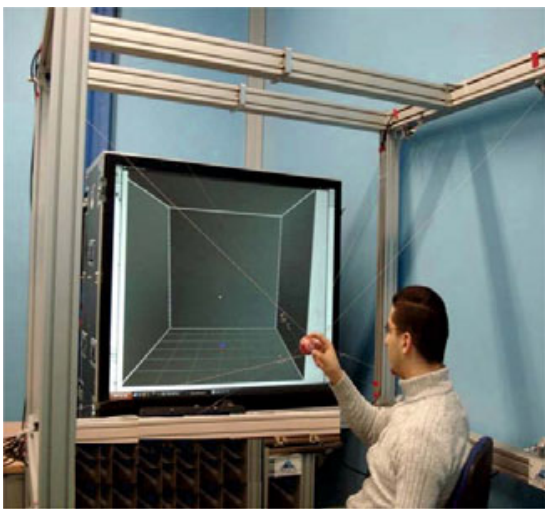

(b)

Fig. 3. Illustration of the dynamic haptic interaction in distributed CVE (a) User 1 with SPIDAR-GH of the semi-immersive platform (b) user 2 with SPIDAR-GM

and SPIDAR-GM means that both of them are SPIDAR-Gs (6DoF) but one is Human scale $(\mathrm{H})$ and the other is Medium scale $(\mathrm{M})$.

\section{Evaluation}

In this section we present the user study carried out to evaluate the effectiveness of our proposed guide.

\subsection{Method}

In order to evaluate the system, twenty volunteers participated in the experiment. They were master and PhD students and had ages from 22 to 30. Majority of them had no prior experience of the use of a haptic device. They all performed the experiment with the same expert. We installed our application on two machines (one for the expert and other for subjects) connected through local area network. Both machines were equiped with a phantom omni. Once the application was launched and the network connection successfully established between the two computers. Two sphers (red and blue) could be seen on both the screens. The red and blue sphers were assigned to the expert and subject respectively. The subjects were required to bring their sphere closed to the expert's sphere when the latter changes its color (i.e becomes green). In addition each subjects was asked that when the force starts guiding his/her hand, then he/she should not look to the screen, in order to avoid learning through visual movements of the cursor (sphere). On the termination of the guiding force he/she was required to wirte the alphabet or geometrical form on a paper to which he/she thinks his/her hand's movement correspond to. There were two guiding sessions. In the first session the subjects were guided to wirte ten alphabets $(\mathrm{L}, \mathrm{G}, \mathrm{M}, \mathrm{R}, \mathrm{S}, \mathrm{P}$, $\mathrm{Z}, \mathrm{W}, \mathrm{B}, \mathrm{N}$ ). In the second session they got guidence for four forms (triangle, square, circle and star). At the end they also responded to a questionnaire. 
Table 1. User perception of the alphabets through haptic guide

\begin{tabular}{|l|c|c|c|c|c|c|c|c|c|c|}
\hline Alphabets & $\mathrm{L}$ & $\mathrm{G}$ & $\mathrm{M}$ & $\mathrm{R}$ & $\mathrm{S}$ & $\mathrm{P}$ & $\mathrm{Z}$ & $\mathrm{W}$ & $\mathrm{B}$ & $\mathrm{N}$ \\
\hline $\begin{array}{l}\text { \% of correct } \\
\text { responses }\end{array}$ & 100 & 90 & 100 & 85 & 95 & 90 & 85 & 80 & 100 & 100 \\
\hline
\end{tabular}

Table 2. User perception of the Forms through haptic guide

\begin{tabular}{|l|c|c|c|c|}
\hline Forms & Triangle & Square & Circle & Star \\
\hline $\begin{array}{l}\% \text { of correct } \\
\text { responses }\end{array}$ & 100 & 100 & 100 & 90 \\
\hline
\end{tabular}

\subsection{Results Analysis}

The table 1 depicts the percentage of correct and incorrect responses in case of guidence for alphabets. Here we see the overall percentage of correct response is high but still there are some subjects that did not perceive correctly some alphabets. This misperception was mainly due to the difference in the method of writing of a character by the expert and subjects. Similarly the table 2 shows that the first three geometrical forms were correctly responded by all subjects, only $10 \%$ subjects percieved the star incorrectly.

In response to the questionnaire, majority of the users were enthusiestic and reported that they found the experience very interesting. Similary we asked them to mark the scale (1-2-3-4-5) for the level of guidence that they were provided. Here $1=$ small level of guidence and $5=$ high level of guidence. The average response was 3.84 (std: 0.68).

\section{Conclusion}

We present a new concept of "What You Feel Is What I Do"(WYFIWID). The concept is fundamentally based on a haptic guide that allows an expert to control the hand of a remot trainee. When haptic guide is active then all movements of the expert's hand (via input device) in the 3D space are haptically reproduced by the trainee's hand via a force feedback device. We used haptic guide to control the trainee's hand for writing alphabets and drawing geometrical forms. Analysing the results of experiments we observed that the users found the haptic guidence very effective and interesting. This guide can be used to teach the writing of alphabets and digits of various languages and drawings to the childern.

We plan to use and extend the concept of WYFIWID in the context of the DigitalOcean project to allow human-robot-interaction in mixed reality environments. In this case the user can feel the ROV (Remotely Operated underwater Vehicle) movements during the exploration mission.

\section{Acknowledgements}

This work is supported by the Conseil Général de l'Essonne, the IBISC laboratory and the DigitalOcean project. 


\section{References}

1. Basdogan, C., Ho, C.-H., Srinivasan, M.A., Slater, M.: Virtual training for a manual assembly task. In: Haptics-e, vol. 2 (2001)

2. Chan, A., Maclean, K., Mcgrenere, J.: Designing haptic icons to support collaborative turn-taking. International Journal of Human-Computer Studies (2007)

3. David, M., Arnaldi, B., Plouzeau, N.: A general framework for cooperative manipulation in virtual environments. In: Virtual Environments 1999 Proceedings of the Eurographics Workshop, pp. 169-178 (1999)

4. Jordan, J., Mortensen, J., Oliveira, M., Slater, M., Tay, B.K., Kim, J., Srinivasan, M.A.: Collaboration in a mediated haptic environment. In: The 5th Annual International Workshop on Presence (2002)

5. Mortensen, J., Vinayagamoorthy, V., Slater, M., Steed, A., Lok, B., Whitton, M.C.: Collaboration in tele-immersive environments. In: Proceedings of the Workshop on Virtual Environments, pp. 93-101 (2002)

6. Otto, O., Roberts, D., Wolff, R.: A review on effective closely-coupled collaboration using immersive cve's. In: VRCIA 2006: Proceedings of the 2006 ACM International Conference on Virtual Reality Continuum and its Applications, pp. 145-154. ACM, New York (2006)

7. Plimmer, B., Crossan, A., Brewster, S.A., Blagojevic, R.: Collaborative virtual sculpting with haptic feedback. In: Virtual Reality, vol. 10, pp. 73-83. Springer, London (2006)

8. Plimmer, B., Crossan, A., Brewster, S.A., Blagojevic, R.: Multimodal collaborative handwriting training for visually-impaired people. In: CHI 2008: Proceeding of the Twenty-sixth Annual SIGCHI Conference on Human Factors in Computing Systems, pp. 393-402. ACM, New York (2008)

9. Prada, R., Payandeh, S.: On study of design and implementation of virtual fixtures. Virtual Reality 13(2), 117-129 (2009)

10. Riva, G., Bacchetta, M., Cesa, G., Molinari, E.: Cybertherapy: Internet and virtual reality as assessment and rehabilitation tools for clinical psychology and neuroscience, pp. 121-164. IOS Press, Amsterdam (2004)

11. Sallnas, E.-L., Rassmus-Grohn, K., Sjostrom, C.: Supporting presence in collaborative environments by haptic force feedback. ACM Trans. Comput.-Hum. Interact. $7(4), 461-476(2000)$

12. Otmane, S., Mallem, M., Kheddar, A., Chavand, F.: Active virtual guide as an apparatus for augmented reality based telemanipulation system on the internet, pp. 185-191. IEEE Computer Society, Los Alamitos (2000)

13. Ullah, S., Ouramdane, N., Otmane, S., Richard, P., Davesne, F., Mallem, M.: Augmenting $3 \mathrm{~d}$ interactions with haptic guide in a large scale virtual environment. The International Journal of Virtual Reality 8, 25-30 (2009)

14. Ullah, S., Richard, P., Otman, S., Mallem, M.: The Effect of audio and Visual aids on task performance in Distributed Collaborative Virtual Environments. In: Intelligent Systems and automation: 2nd Mediterranean Conference on Intelligent Systems and Automation (CISA 2009). AIP Conference Proceedings, vol. 1107, pp. 196-201 (2009)

15. Oakley, I., Brewster, S., Gray, P.: Can You Feel the Force? An Investigation of Haptic Collaboration in Shared Editors. In: Proceedings of Eurohaptics, pp. 54-59 (2001) 
16. Feygin, D., Keehner, M., Tendick, R.: Haptic guidance: experimental evaluation of a haptic training method for a perceptual motor skill. In: Proceedings of the 10th Symposium on Haptic Interfaces for Virtual Environment and Teleoperator Systems, pp. 40-47 (2002)

17. Yoshikawa, T., Henmi, K.: Human skill transfer using haptic virtual reality technology. In: The 6th International Symposium on Experimental Robotics, vol. 250, pp. 351-360 (2000)

18. Dan, M., Hong, T., Federico, B., Timothy, C., Kenneth, S.: Haptic Feedback Enhances Force Skill Learning. In: Proceedings of the Second Joint EuroHaptics Conference and Symposium on Haptic Interfaces for Virtual Environment and Teleoperator Systems, pp. 21-26 (2007)

19. Bluteau, J., Gentaz, E., Coquillart, S., Payan, Y.: Haptic guidances increase the visuo-manual tracking of Japanese and Arabic letters. In: International Multisensory Research Forum (2008)

20. Chellali, A., Dumas, C., Milleville, I.: WYFIWIF: A Haptic Communication Paradigm For Collaborative Motor Skills Learning. In: Proceedings of the Web Virtual Reality and Three-Dimensional Worlds (2010)

21. Makoto, S.: Development of String-based Force Display: SPIDAR. In: IEEE VR Conference (2002) 